\title{
On Control of a Boost DC-DC Power Converter under Constrained Input
}

\author{
Javier Moreno-Valenzuela and Octavio García-Alarcón \\ Instituto Politécnico Nacional-CITEDI, Ave. Instituto Politécnico Nacional 1310, Nueva Tijuana, 22435 Tijuana, BC, Mexico \\ Correspondence should be addressed to Javier Moreno-Valenzuela; moreno@citedi.mx
}

Received 30 July 2016; Revised 14 November 2016; Accepted 6 December 2016; Published 15 January 2017

Academic Editor: Alfred Hubler

Copyright (c) 2017 J. Moreno-Valenzuela and O. García-Alarcón. This is an open access article distributed under the Creative Commons Attribution License, which permits unrestricted use, distribution, and reproduction in any medium, provided the original work is properly cited.

\begin{abstract}
In this paper, a new controller for a boost DC-DC (direct current to direct current) power converter is proposed. The discussed DCDC boost converter model considers the losses coming from the inductor and capacitor. The novel control scheme takes into account that the duty cycle is constrained to physically admissible values. The analysis of the closed-loop trajectories provides the conclusion that output voltage regulation is achieved in asymptotic form. In addition, the problem of uncertain supply voltage and unmeasurable inductor current is also addressed by using an observer together with the proposed control law. Our theoretical results are supported by using numerical simulations and experimental tests. Comparisons with respect to known approaches are presented.
\end{abstract}

\section{Introduction}

The voltage of many electrical and electronic systems is often higher than the voltage of the main source, for example, in systems powered by batteries. A conventional solution employs the so called boost DC-DC (direct current to direct current) converter where the increase of the output voltage is accomplished. Textbooks giving introduction to the history, construction, and control of the boost power converters are [1-4].

The boost power converter is applied in photovoltaic systems [5], mobile communication circuits [6], power factor correction [7], and hybrid electric vehicle systems [8]. This power converter is a bilinear second order nonminimum phase system and under certain operation conditions can be affected by disturbances and other nonlinearities.

The perspective in power electronics engineering to control a boost power converter relies on the characterization of the devices and in the design of compensation circuits. On the other hand, in control engineering, the approach to regulate the output voltage is to modify the duty cycle, thus compensating the losses due to the operation of the components.

In order to provide a degree of robustness to compensate uncertainties in the load, supply voltage, and unmodeled disturbances, many control algorithms have been devised to achieve voltage output regulation; see, for example, [9-12]. More recently, the work in [13] presented a comparison of nonlinear controllers for the DC-DC boost power converter. In [14], the problem of output feedback regulation via Lyapunov's theory was addressed.

Usually, the duty cycle percentage is the control input for the boost power converter. This a number that is into the set $[0,1)$ and this constraint is rarely taken into account to design a control system. The reason probably is the increasing in the complexity of the closed-loop system stability analysis. As pointed out in the textbook [15, p. 171]: "saturation is probably the most commonly encountered nonlinearity in control engineering."

Works considering saturation of duty cycle in control input for buck converters can be found in $[16,17]$. Specifically, the research in [16] used a LMI approach supported by simulation results. Paper [17] presented a comparison between two controllers and included a discussion of stability analysis. More recently, in the work in [18], an adaptive neural network controller is introduced. However, this scheme is based on the concept of inverting a saturation function, which is not globally possible. More recently, in [19], the problem of robust stability and tracking of a saturated control buck DC-DC converter was considered. There, LMIs were used to insert the constraints in the design phase while imposing positivity 
in the closed-loop state. Other problems with saturation in the dynamics (not in the system input) have been addressed in, for example, [20], where a nonlinear controller with an inherent current limiting capability was presented for different types of DC-DC power converters.

There have been only a few works that address the problem of voltage regulation for boost converters control under constrained duty cycle percentage. For example, in [21] a model-based full state-feedback controller was introduced. The efficiency of the controller was proven by real-time experiments. A more sophisticated scheme was given by Karagiannis et al. [22] addressing the problem of controlling the boost converter by using only output voltage measurement while the supply voltage is unknown. More recently, in [23] a saturated state-feedback control law for a batterydriven boost converter was introduced. In [24], a control design procedure for boost power converters was reported, and, although this procedure ensures robustness for the supply voltage and output load variations, input saturation is avoided.

Many of the controllers reported in literature do not take into account the parasitic resistance in the inductor and capacitor, including the works cited earlier. Although the parasitic resistances are relatively small, they cannot be ignored in the practical DC-DC boost converter because it increases the model uncertainty. The proposed controller in this paper is designed on the basis that the losses due to parasitic resistances are present.

The contributions of this paper are the following. Firstly, we introduce a new controller for an input-constraint boost power converter. We prove asymptotic convergence of the output voltage error in spite of the fact that the system is affected by input saturation. Secondly, an observer for the supply voltage is revisited. The implications of using this new observer together with new controller are studied. A simulation study complements the theoretical results, where comparisons are given. Besides, a real-time experimental study supports the practical viability of the proposed scheme.

Better results are obtained with the new controller.

The present document is organized as follows. Section 2 deals with the modeling of DC-DC boost converters and the control goal. In Section 3, the new controller is introduced. The implications of using an observer to estimate the supply voltage with the new controller are discussed in Section 4 . The simulation results are presented in Section 5. Experimental results are given in Section 6. Finally, Section 7 presents some concluding remarks.

\section{Boost DC-DC Power Converter Model and Control Goal}

2.1. Boost DC-DC Power Converter Model. This paper deals with the output voltage regulation problem of the DC-DC boost converter shown in Figure 1. We consider a practical inductor with a parasitic resistance. This model also includes a resistor to represent unavoidable loss, which dissipates power as the capacitor is charged or discharged. The nominal values of the resistors are assumed to be known. By using

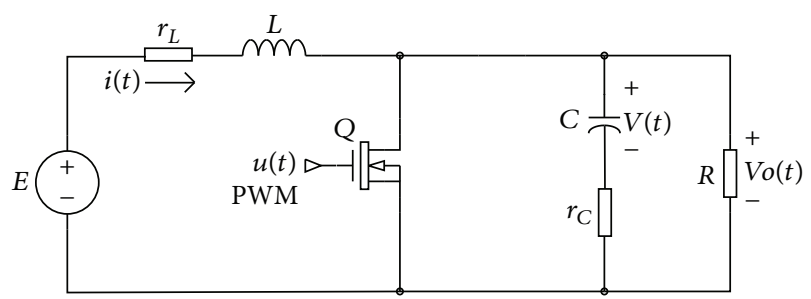

FIGURE 1: The boost converter circuit having parasitic resistances $r_{L}$ and $r_{C}$.

an average switching method, the mathematical model of Figure 1 is described by $[2,3]$

$$
\begin{aligned}
L \frac{d i}{d t}= & -\left[r_{L}+[1-u]^{2} \frac{r_{C} R}{r_{C}+R}\right] i-[1-u] \frac{r_{C} R}{r_{C}+R} v \\
& +E \\
C \frac{d v}{d t}= & {[1-u] \frac{R}{r_{C}+R} i-\frac{v}{r_{C}+R}, }
\end{aligned}
$$

where $u(t)$ is a continuous control signal representing the duty cycle percentage of the PWM circuit controlling the switch, the positive quantity $E$ represents the external voltage supply, $i(t)$ is the current through inductor $L, v(t)$ is the voltage through capacitor $C$, and $r_{L}, r_{C}$, and $R$ denote the inductance, the capacitance, and the load resistance, respectively.

The output voltage is given by

$$
v_{o}=\frac{R}{r_{C}+R} v+[1-u] \frac{r_{C} R}{r_{C}+R} i .
$$

See the textbooks [2-4] for further details in the modeling of the boost DC-DC converter.

2.2. Control Goal. An equilibrium point of system (1), assuming a constant duty cycle percentage

$$
u(t)=1-D^{*}
$$

satisfies

$$
\begin{aligned}
& 0=-\left[r_{L}+D^{* 2} \frac{r_{C} R}{r_{C}+R}\right] i_{d}-D^{*} \frac{r_{C} R}{r_{C}+R} v_{d}+E, \\
& 0=D^{*} \frac{R}{r_{C}+R} i_{d}-\frac{v_{d}}{r_{C}+R},
\end{aligned}
$$

which has solution

$$
\begin{aligned}
& i_{d}=\frac{v_{d}}{D^{*} R}, \\
& v_{d}=\frac{D^{*} R E}{r_{L}-D^{* 2} R} .
\end{aligned}
$$

Since $v_{d}$ is an independent parameter, (6) can be solved for $D^{*}$, which is the duty cycle complement. Hence

$$
D^{*}=D_{1}^{*} \text { or } D_{2}^{*} \text {, }
$$


where

$$
\begin{aligned}
& D_{1}^{*}=\frac{R E+\sqrt{[R E]^{2}-4 R v_{d}^{2} r_{L}}}{2 R v_{d}}, \\
& D_{2}^{*}=\frac{R E-\sqrt{[R E]^{2}-4 R v_{d}^{2} r_{L}}}{2 R v_{d}},
\end{aligned}
$$

where $v_{d}$ is the desired capacitor voltage. Throughout this paper, the solution $D^{*}=D_{1}^{*}$ is considered.

An important observation is that, at the operation point $i=i_{d}$ and $v=v_{d}$, the output voltage is given by $v_{o}=v_{d}$, which can be confirmed from the definition of $v_{o}$ in (2). Hence, $v_{d}$ denotes the desired output voltage.

In general, we can say that $D^{*}$ is a function of the load resistance, input voltage, the desired voltage, and inductor resistance; that is,

$$
D^{*}\left(R, E, v_{d}, r_{L}\right) .
$$

In practice, the actual duty cycle percentage $u(t)$ should satisfy the constraint

$$
0<u(t)<1, \quad \forall t \geq 0,
$$

whereby at the equilibrium point (3) should also satisfy

$$
0<1-D^{*}\left(R, E, v_{d}, r_{L}\right)<1 .
$$

This means that, for fixed parameters $R, E$, and $r_{L}$, the desired voltage should be selected so that (8) satisfies restriction (11), which is always guaranteed for

$$
v_{d}>\frac{R}{R+r_{L}} E .
$$

The control goal consists in the specification of the desired output voltage $v_{d}$ satisfying (12) and the design of a control law $u(t)$ satisfying constraint (10) such that the output voltage in (2) achieves

$$
\lim _{t \rightarrow \infty} v_{o}(t)=v_{d},
$$

while the inductor current $i(t)$ and capacitor voltage $v(t)$ remain bounded.

\section{Proposed Controller}

In this section, a controller is introduced so that constraint

(10) is accomplished.

Let us define the saturation function

$$
\operatorname{sat}(\bar{u})= \begin{cases}u_{\max }, & \text { for } \bar{u}>u_{\max }, \\ \bar{u}, & \text { for } u_{\min } \leq \bar{u} \leq u_{\max }, \\ u_{\min }, & \text { for } \bar{u}<u_{\min },\end{cases}
$$

with the inequality

$$
0<u_{\min }<D^{*}<u_{\max }<1
$$

being satisfied. Therefore,

$$
\operatorname{sat}\left(D^{*}\right)=D^{*} .
$$

The proposed controller is given by

$$
\begin{aligned}
& u=1-\operatorname{sat}\left(D^{*}+\phi\right), \\
& \dot{\phi}=\gamma\left[v_{d} e_{i}-i_{d} e_{v}\right]-\gamma k_{a w}\left[\operatorname{sat}\left(D^{*}+\phi\right)-D^{*}\right],
\end{aligned}
$$

where $\gamma$ and $k_{a w}$ are strictly positive constants, and

$$
\begin{gathered}
e_{i}=i-i_{d}, \\
e_{v}=v-v_{d},
\end{gathered}
$$

which denote the inductor current error and the capacitance voltage error, respectively.

The definition of the saturation function in (14) allows guaranteeing that $u(t)$ actually satisfies (10); that is,

$$
1-u_{\max } \leq u(t) \leq 1-u_{\min },
$$

so that

$$
0<1-u_{\max }<D^{*}<1-u_{\min }<1 .
$$

By defining

$$
\delta=\bar{u}-\operatorname{sat}(\bar{u}),
$$

with

$$
\bar{u}=D^{*}+\phi,
$$

and substituting the control action (17) into the boost converter equations (1), the error dynamics can be written as

$$
\begin{aligned}
L \frac{d e_{i}}{d t}= & \delta \frac{R}{r_{C}+R} v-\left[r_{L}+\operatorname{sat}(\bar{u})^{2} \frac{r_{C} R}{r_{C}+R}\right] e_{i}-r_{L} i_{d} \\
& -\operatorname{sat}(\bar{u})^{2} \frac{r_{c} R}{r_{C}+R} i_{d}+D^{* 2} \frac{r_{C} R}{r_{C}+R} i_{d} \\
& -D^{*} \frac{r_{C} R}{r_{C}+R} i_{d}+E \\
& -\left[\left[D^{*}+\phi\right] e_{v}+v_{d} \phi+D^{*} v_{d}\right] \frac{R}{r_{C}+R}, \\
C \frac{d e_{v}}{d t}= & -\delta \frac{R}{r_{C}+R} i-\frac{e_{v}}{r_{C}+R}-\frac{v_{d}}{r_{C}+R} \\
& +\left[D^{*}+\phi\right] \frac{R}{r_{C}+R} e_{i}+\phi \frac{R}{r_{C}+R} i_{d} \\
& +D^{*} \frac{R}{r_{C}+R} i_{d}
\end{aligned}
$$

with $\delta$ in (23).

It is noteworthy to say that

$$
0=-r_{L}-D^{*} \frac{r_{C} R}{r_{C}+R} i_{d}+E-D^{*} v_{d} \frac{R}{r_{C}+R},
$$

$$
0=D^{*} \frac{R}{r_{\mathrm{C}}+R} i_{d}-\frac{v_{d}}{r_{\mathrm{C}}+R} \text {, }
$$


which correspond to the boost power converter equations (1) evaluated at the operation point $i=i_{d}, v=v_{d}$, and $u=1-D^{*}$.

Using (26) into (25), the overall closed-loop system can be written as

$$
\begin{aligned}
L \frac{d e_{i}}{d t}= & \delta \frac{R}{r_{C}+R} v-\left[r_{L}+\operatorname{sat}(\bar{u})^{2} \frac{r_{C} R}{r_{C}+R}\right] e_{i} \\
& +\left[D^{* 2}-\operatorname{sat}(\bar{u})^{2}\right] \frac{r_{c} R}{r_{C}+R} i_{d} \\
& -\left[D^{*}+\phi\right] e_{v} \frac{R}{r_{C}+R}-v_{d} \phi \frac{R}{r_{C}+R}, \\
C \frac{d e_{v}}{d t}= & {\left[D^{*}+\phi\right] \frac{R}{r_{C}+R} e_{i}+\phi \frac{R}{r_{C}+R} i_{d}-\frac{e_{v}}{r_{C}+R} } \\
& -\delta \frac{R}{r_{C}+R} i, \\
\frac{d}{d t} \phi= & \gamma\left[v_{d} e_{i}-i_{d} e_{v}\right]-\gamma k_{a w}\left[\operatorname{sat}\left(D^{*}+\phi\right)-D^{*}\right] .
\end{aligned}
$$

It is possible to show that the state space origin

$$
\left[\begin{array}{l}
e_{i} \\
e_{v} \\
\phi
\end{array}\right]=\left[\begin{array}{l}
0 \\
0 \\
0
\end{array}\right] \in \mathbb{R}^{3}
$$

is an equilibrium point of the closed-loop system (27).

The following Lemma will be valuable in the coming analysis.

Lemma 1. The function

$$
\mathcal{U}(\phi)=\frac{\phi^{2}}{2}-\int_{0}^{\phi} \delta(\bar{u}) d \phi
$$

is positive definite.

Proof. First, note that $\bar{u}$ in (24) is a function of $\phi$. Then, by direct integration

$$
\begin{aligned}
& \int_{0}^{\phi} \delta(\bar{u}) d \phi \\
& \quad= \begin{cases}f^{-}(\phi), & \text { for } \phi<u_{\min }-D^{*} \\
0, & \text { for } u_{\min }-D^{*} \leq \phi \leq u_{\max }-D^{*} \\
f^{+}(\phi), & \text { for } \phi>u_{\max }-D^{*}\end{cases}
\end{aligned}
$$

where

$$
\begin{aligned}
& f^{-}(\phi)=\frac{1}{2} \phi^{2}-\left[u_{\min }-D^{*}\right] \phi+\frac{1}{2}\left[u_{\min }-D^{*}\right]^{2}, \\
& f^{+}(\phi)=\frac{1}{2} \phi^{2}-\left[u_{\max }-D^{*}\right] \phi+\frac{1}{2}\left[u_{\max }-D^{*}\right]^{2} .
\end{aligned}
$$

The superscripts “-” and "+" are related to the sectors where $\phi<0$ and $\phi>0$, respectively.
By substituting (30) into (29)

$\mathcal{U}(\phi)$

$$
= \begin{cases}\mathcal{U}^{-}(\phi), & \text { for } \phi<u_{\min }-D^{*}, \\ \frac{1}{2} \phi^{2}, & \text { for } u_{\min }-D^{*} \leq \phi \leq u_{\max }-D^{*}, \\ \mathcal{U}^{+}(\phi), & \text { for } \phi>u_{\max }-D^{*},\end{cases}
$$

where

$$
\begin{aligned}
& \mathcal{U}^{-}(\phi)=\left[u_{\min }-D^{*}\right] \phi-\frac{1}{2}\left[u_{\min }-D^{*}\right]^{2}, \\
& \mathcal{U}^{+}(\phi)=\left[u_{\max }-D^{*}\right] \phi-\frac{1}{2}\left[u_{\max }-D^{*}\right]^{2} .
\end{aligned}
$$

By noticing that $\mathcal{U}^{-}(\phi)>0$ for $\phi>u_{\max }-D^{*}$ and $\mathcal{U}^{-}(\phi)>0$ for $\phi<u_{\text {min }}-D^{*}$ the proof of Lemma 1 is completed.

Proposition 2. The state space origin of the closed-loop system (27) is globally asymptotically stable if

$$
k_{a w}>\frac{\left[\left[3 D^{*}+u_{\max }\right]\left(r_{C} R /\left(r_{C}+R\right)\right) i_{d}\right]^{2}}{4\left[r_{L}+u_{\min }^{2}\left(r_{C} R /\left(r_{C}+R\right)\right)\right]}
$$

is satisfied.

Proof. In order to show global stability of the state space origin the following Lyapunov function candidate is proposed:

$$
\mathscr{W}\left(e_{i}, e_{v}, \phi\right)=\frac{L}{2} e_{i}^{2}+\frac{C}{2} e_{v}^{2}+\gamma^{-1} \frac{R}{r_{C}+R} \mathscr{U}(\phi),
$$

with $\gamma>0$ and $\mathscr{U}(\phi)$ is defined in (29). The function $\mathscr{W}$ in (35) is globally positive definite and radially unbounded.

By using the facts

$$
\begin{aligned}
v_{d} e_{i}-i_{d} e_{v} & =v e_{i}-i e_{v}, \\
\text { sat }(\bar{u})-D^{*} & =\phi-\delta(\bar{u}),
\end{aligned}
$$

where $e_{i}$ and $e_{v}$ were defined in (19) and (20), respectively, and $\bar{u}=D^{*}+\phi$ was defined in (24), it is possible to prove that the time derivative of $\mathscr{W}$ along the closed-loop system (27) is given by

$$
\begin{aligned}
\dot{\mathscr{W}}= & -\left[r_{L}+\operatorname{sat}(\bar{u})^{2} \frac{r_{C} R}{r_{C}+R}\right] e_{i}^{2} \\
& +\left[D^{* 2}-\operatorname{sat}(\bar{u})^{2}\right] \frac{r_{C} R}{r_{C}+R} i_{d} e_{i}-\frac{e_{v}^{2}}{r_{C}+R} \\
& -k_{a w}[\phi-\delta(\bar{u})]^{2} .
\end{aligned}
$$

After some algebra, it is possible to show that

$$
[\phi-\delta]^{2}=\operatorname{sat}(\bar{u})^{2}-D^{* 2}+2 D^{*}-2 \operatorname{sat}(\bar{u}) D^{*},
$$

from which

$$
D^{* 2}-\operatorname{sat}(\bar{u})^{2}=-[\phi-\delta]^{2}-2 D^{*}[\phi-\delta] .
$$


Equation (39) can be used to rewrite $\dot{\mathscr{W}}$ as follows:

$$
\begin{aligned}
\dot{\mathscr{V}}= & -\left[r_{L}+\operatorname{sat}(\bar{u})^{2} \frac{r_{C} R}{r_{C}+R}\right] e_{i}^{2} \\
& -[\phi-\delta]^{2} \frac{r_{C} R}{r_{C}+R} i_{d} e_{i} \\
& -2 D^{*}[\phi-\delta] \frac{r_{C} R}{r_{C}+R} i_{d} e_{i}-\frac{e_{v}^{2}}{r_{C}+R} \\
& -k_{a w}[\phi-\delta(\bar{u})]^{2} .
\end{aligned}
$$

Besides, it should be noticed that

$$
|\phi-\delta|=\left|D^{*}-\operatorname{sat}(\bar{u})\right| \leq D^{*}+u_{\max },
$$

which allows obtaining the upper bound

$$
\begin{gathered}
-[\phi-\delta]^{2} \frac{r_{C} R}{r_{C}+R} i_{d} e_{i}-2 D^{*}[\phi-\delta] \frac{r_{C} R}{r_{C}+R} i_{d} e_{i} \\
\leq\left[3 D^{*}+u_{\max }\right] \frac{r_{C} R}{r_{C}+R} i_{d}|\phi-\delta|\left|e_{i}\right| .
\end{gathered}
$$

Finally, the following upper bound on $\dot{\mathscr{W}}$ is obtained:

$$
\dot{\mathscr{V}} \leq-\left[\begin{array}{c}
\left|e_{i}\right| \\
|\phi-\delta|
\end{array}\right]^{T} Q\left[\begin{array}{c}
\left|e_{i}\right| \\
|\phi-\delta|
\end{array}\right]-\frac{\left|e_{v}\right|^{2}}{r_{C}+R},
$$

with

$$
=\left[\begin{array}{cc}
r_{L}+u_{\min }^{2} \frac{r_{C} R}{r_{C}+R} & -\frac{1}{2}\left[3 D^{*}+u_{\max }\right] \frac{r_{C} R}{r_{C}+R} i_{d} \\
-\frac{1}{2}\left[3 D^{*}+u_{\max }\right] \frac{r_{C} R}{r_{C}+R} i_{d} & k_{a w}
\end{array}\right] .
$$

A sufficient condition for $\dot{\mathscr{W}}$ in (43) to be globally negative definite is that the symmetric matrix $Q$ be positive definite, which is always satisfied for condition (34). Therefore, there are sufficient conditions to ensure that the state-space origin of the closed-loop system (27) is globally uniformly asymptotically stable; see, for example, [25, 26], which at the same time implies

$$
\lim _{t \rightarrow \infty}\left[\begin{array}{l}
e_{i}(t) \\
e_{v}(t) \\
\phi(t)
\end{array}\right]=\left[\begin{array}{l}
0 \\
0 \\
0
\end{array}\right],
$$

for all initial conditions $\left[\begin{array}{lll}e_{i}(0) & e_{v}(0) & \phi(0)\end{array}\right]^{T} \in \mathbb{R}^{3}$.

It is noteworthy to say that derived from the proof of Proposition 2 the condition for ensuring global stability of the closed-loop system (27) is to increase the numerical value of the gain $k_{a w}$. The numerical simulation and the realtime experimental evaluation have taken into account such a condition; that is, a large value of the gain $k_{a w}$ has been used.
On the other hand, limit (45) implies that control goal (13) is satisfied, which is ensured by the continuity of the output voltage function $v_{o}(t)$ in (2) and by the fact that

$$
v_{o}(i, v)=v_{d}, \quad \text { for } i=i_{d}, v=v_{d},
$$

which was mentioned earlier.

Proposition 2 provides the theoretical framework for controller (17) and (18) to accomplish output voltage regulation under the constrained control input; that is, the duty cycle percentage remains in the physical admissible limit for all time.

Another interesting observation is that the first bracketed term of the dynamics of $\phi(t)$ in (18) is a stabilization term while the second one corresponds to an antiwindup term, which helps to increase the rate of convergence of the voltage error when the input saturation is activated.

A way to generalize the proposed design in (17) and (18) is by considering that $\phi(t)$ is a signal computed as the integral of

$$
\dot{\phi}=\Phi\left(e_{i}, e_{v}, \phi\right),
$$

where $\Phi\left(e_{i}, e_{v}, \phi\right)$ is a continuous function satisfying the conditions

(i) $\Phi(0,0,0)=0$,

(ii) $\dot{\mathscr{V}} \leq 0$ for all $\left[\begin{array}{lll}e_{i} & e_{v} & \phi\end{array}\right]^{T} \in \mathbb{R}^{3}$, which can also be achieved by defining $k_{a w}$ as a time-varying or statedependent gain, for example, $k_{a w}\left(e_{i}(t), e_{v}(t)\right)$.

In other words, the function $\Phi\left(e_{i}, e_{v}, \phi\right)$ can be designed to shape the energy of the Lyapunov function $\mathscr{W}$.

\section{Proposed Controller under Uncertain Input Voltage $E$ and Unmeasured Current $i(t)$}

The proposed controller (17) and (18) has the following properties:

(i) It is able to increase the convergence rate of the output voltage error.

(ii) It respects the physical limit of duty cycle percentage.

(iii) It is equipped with an antiwindup term (the one associated with the gain $k_{a w}$ ).

However, the proposed scheme is based on the assumption that all parameters are known. In practice, this assumption may not be satisfied. Therefore, the addition of a degree of robustness strengthens the properties that the controller already possesses.

Let us consider that there are available signals $\widehat{E}(t)$ and $\hat{i}(t)$, which are estimations of the input voltage $E$ and the actual inductor current $i(t)$, respectively. The new controller (17) and (18) written in terms of signals $\widehat{E}(t)$ and $\widehat{i}(t)$ is given by

$$
\begin{aligned}
u= & 1-\operatorname{sat}\left(\widehat{D}^{*}+\phi\right), \\
\dot{\phi}= & \gamma\left[v_{d}\left[\hat{i}-i_{d}^{*}\right]-i_{d}^{*} e_{v}\right] \\
& \quad-\gamma k_{a w}\left[\operatorname{sat}\left(\widehat{D}^{*}+\phi\right)-\widehat{D}^{*}\right],
\end{aligned}
$$


TABLE 1: Parameters of the boost converter.

\begin{tabular}{lcc}
\hline Symbol & Value & Unit \\
\hline$L$ & 150 & $\mathrm{mH}$ \\
$C$ & 1000 & $\mu \mathrm{F}$ \\
$R$ & 100 & $\Omega$ \\
$r_{L}$ & 0.9 & $\Omega$ \\
$r_{C}$ & 0.4 & $\Omega$ \\
\hline
\end{tabular}

where

$$
\begin{aligned}
& \widehat{D}^{*}=\widehat{D}_{1}^{*}=\frac{R \widehat{E}+\sqrt{[R \widehat{E}]^{2}-4 R v_{d}^{2} r_{L}}}{2 R v_{d}}, \\
& i_{d}^{*}=\frac{v_{d}^{2}}{\widehat{E} R}
\end{aligned}
$$

are the estimated duty cycle complement and the estimated desired current, respectively. In order to avoid singularity, we assume that $\widehat{E}(t)>0$ and $[R \widehat{E}(t)]^{2}-4 R v_{d}^{2} r_{L}>0$ for all $t \geq 0$.

Let us define

$$
\mathbf{z}=\left[\begin{array}{c}
\widetilde{E} \\
\widetilde{i}
\end{array}\right]=\left[\begin{array}{c}
\widehat{E} \\
\hat{i}
\end{array}\right]-\left[\begin{array}{c}
E \\
i
\end{array}\right],
$$

which denotes the observation error whose dynamics are globally asymptotically stable.

In Section 6, where experimental results are given, an observer-based controller already proposed in the literature is revisited. This design is used as inspiration to introduce a new observer-based controller, which is defined by (48), and an observer given in literature.

It is possible to show that the overall electrical and observation error dynamics can be written in the compact form:

$$
\begin{aligned}
\frac{d}{d t} \mathbf{e} & =\mathbf{f}_{E}(t, \mathbf{e})+G(t, \mathbf{e}, \mathbf{z}) \boldsymbol{\alpha}(t, \mathbf{e}, \mathbf{z}), \\
\frac{d}{d t} \mathbf{z} & =\mathbf{f}_{O}(t, \mathbf{z})
\end{aligned}
$$

where $\mathbf{e}=\left[\begin{array}{ll}e_{i} & e_{v}\end{array}\right]^{T} \in \mathbb{R}^{2}$ and $\mathbf{z}=\left[\begin{array}{ll}\widetilde{E} & \widetilde{i}\end{array}\right]^{T} \in \mathbb{R}^{2}$.

In fact, the observer to be used in the real-time experiments presents error independent dynamics as shown in (52), where $\mathbf{z}=0$ is the only equilibrium point.

Besides, it is also possible to prove that the state space origin $\left[\begin{array}{ll}\mathbf{e}^{T} & \mathbf{z}^{T}\end{array}\right]^{T}=0$ is an equilibrium point of (51) and (52). The overall closed-loop system (51) and (52) has the structure of a cascaded nonlinear system, as the one studied in [27].

For compactness of the paper, we have decided to leave aside the explicit proof of asymptotic stability of the cascaded system (51) and (52) and we will focus on the experimental results.

\section{Simulation Results}

We have performed numerical simulations using the parameters in Table 1. For all simulations, we have assumed the initial

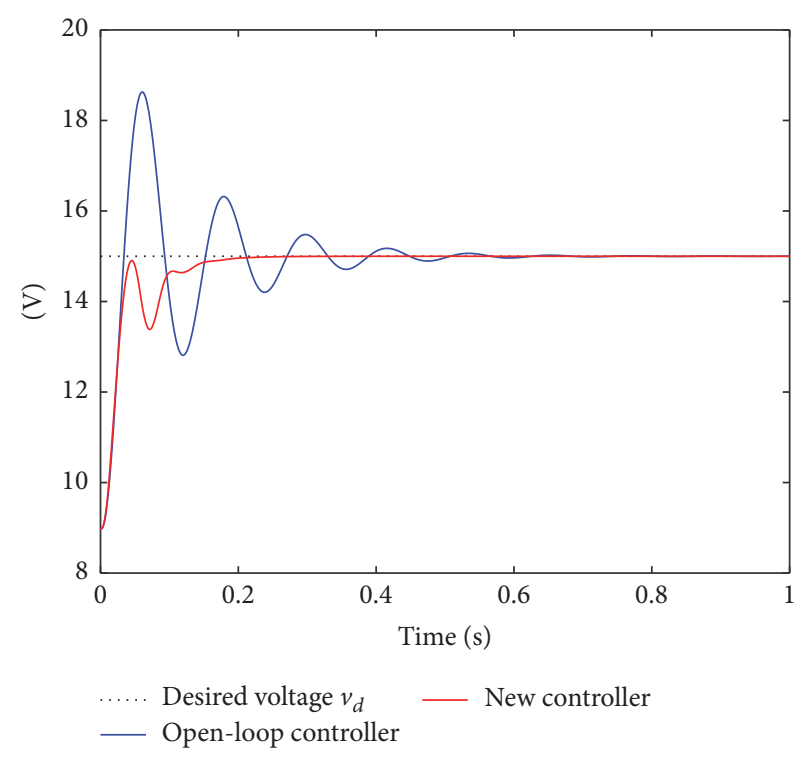

FIGURE 2: Capacitor voltage $v(t)$ obtained for the new controller (17) and (18) and the open-loop controller (53).

conditions $i(0)=0.1[\mathrm{~A}]$ and $v(0)=9[\mathrm{~V}]$ in the boost DCDC power converter.

This case study consists in comparing the new controller (17) and (18) with respect to the open-loop controller

$$
u=1-D^{*} \text {. }
$$

This numerical test also assumes the known constant parameter $E=10[\mathrm{~V}]$, a desired voltage $v_{d}=15[\mathrm{~V}]$, and the initial condition $\phi(0)=0$ for integral action (18). The selected gains for the new controller (17) and (18) are

$$
\begin{aligned}
\gamma & =10, \\
k_{a w} & =10 .
\end{aligned}
$$

The limits for the saturation function are

$$
\begin{aligned}
& u_{\text {min }}=0.2, \\
& u_{\max }=0.8 .
\end{aligned}
$$

Let us notice that the boost power converter parameters, the saturation limits in (55), and the numerical value of the gain $k_{a w}$ in (54) satisfy condition (34) for the global stability of the state space origin of the closed-loop system (27).

The results are in Figure 2, which shows the capacitor voltage $v(t)$ obtained for the new controller (17) and (18) and the open-loop controller (53). There, the superiority of the new scheme is clearly appreciated since the settling time is much shorter. Figure 3 depicts the duty cycle percentage $u(t)$ obtained by using the mentioned control schemes.

\section{Experimental Results}

In this section, we first describe the mathematical characterization of the controllers to be tested and after the experimental results are presented. 


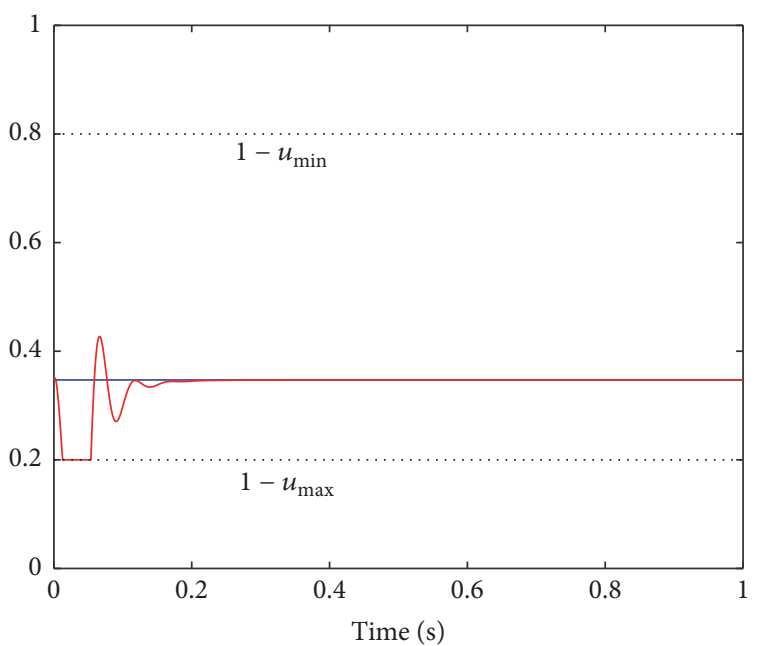

Open-loop controller

- New controller

FIGURE 3: Duty cycle percentage $u(t)$ obtained for the new controller (17) and (18) and the open-loop controller (53).

6.1. Observer-Based Controller Proposed in [22]. In the work [22], a control methodology for the adaptive control of a class of nonlinear systems was used to solve the robust regulation problem for a DC-DC boost converter with partial parameter and state information. In particular, the boost converter model considered there was assumed to be lossless; that is, in model (1) the parameters $r_{L}$ and $r_{C}$ were considered nulls, which also implies that $v_{o}(t)=v(t)$ as can be verified from (2).

The observer-based controller proposed in [22], which will be also referred to as KAO controller by its authors, is given by

$$
u=1-\operatorname{sat}\left(\frac{\widehat{E}}{v_{d}}\right) \text {, }
$$

which is the control input, and the observer

$$
\begin{aligned}
\frac{d}{d t} \eta_{1}= & -\frac{\lambda_{1}}{C}\left[\left[\eta_{2}+\lambda_{2} v\right][1-u]-\frac{1}{R} v\right], \\
\frac{d}{d t} \eta_{2}= & -\frac{\lambda_{2}}{C}\left[\left[\eta_{2}+\lambda_{2} v\right][1-u]-\frac{1}{R} v\right] \\
& +\frac{1}{L}\left[\eta_{1}+\lambda_{1} v-v[1-u]\right]
\end{aligned}
$$

with constants $\lambda_{1}, \lambda_{2}>0$, whose outputs are

$$
\begin{aligned}
\widehat{E}(t) & =\eta_{1}(t)+\lambda_{1} v(t), \\
\widehat{i}(t) & =\eta_{2}(t)+\lambda_{2} v(t) .
\end{aligned}
$$

The signals in (58) denote the estimated supply voltage, which is particularly used in the control input (56), and estimated inductor current, respectively.
By using the boost converter equations in (1) with $r_{L}$ and $r_{C}$ null, the definition of $\mathbf{z}$ in (50), and the observer equations (57) and (58), it is possible to show that

$$
\frac{d}{d t}\left[\begin{array}{c}
z_{1} \\
z_{2}
\end{array}\right]=\left[\begin{array}{c}
-\frac{\lambda_{1}}{C}[1-u] z_{2} \\
\frac{1}{L} z_{1}-\frac{\lambda_{2}}{C}[1-u] z_{2}
\end{array}\right]
$$

whose equilibrium point $\mathbf{z}=0$ is asymptotically stable. Notice that system (59) presents the structure of system (52).

6.2. New Observer-Based Controller. Motivated by the results reported in [22], and using the structure of the controller in (17) and (18), a new observer-based controller was described in (48), which is rewritten here for the sake of reference

$$
\begin{aligned}
u= & -\operatorname{sat}\left(\widehat{D}^{*}+\phi\right), \\
\dot{\phi}= & \gamma\left[v_{d}\left[\widehat{i}-i_{d}^{*}\right]-i_{d}^{*} e_{v}\right] \\
& \quad-\gamma k_{a w}\left[\operatorname{sat}\left(\widehat{D}^{*}+\phi\right)-\widehat{D}^{*}\right],
\end{aligned}
$$

where

$$
\begin{aligned}
\widehat{D}^{*} & =\widehat{D}_{1}^{*}=\frac{R \widehat{E}+\sqrt{[R \widehat{E}]^{2}-4 R v_{d}^{2} r_{L}}}{2 R v_{d}}, \\
i_{d}^{*} & =\frac{v_{d}^{2}}{\widehat{E} R} .
\end{aligned}
$$

Inspired by the structure of the observer proposed in [22], which is given in (57), we propose the following observer:

$$
\begin{aligned}
\frac{d}{d t} \eta_{1}= & -\frac{\lambda_{1}}{C}\left[[1-u] \frac{R}{r_{C}+R} \widehat{i}-\frac{1}{r_{C}+R} v\right], \\
\frac{d}{d t} \eta_{2}= & -\frac{\lambda_{2}}{C}\left[[1-u] \frac{R}{r_{C}+R} \hat{i}-\frac{1}{r_{C}+R} v\right] \\
& +\frac{1}{L}\left[-[1-u] \frac{R}{r_{C}+R} v+\widehat{E}-r \hat{i}\right],
\end{aligned}
$$

with

$$
r=r_{L}+[1-u]^{2} \frac{r_{C} R}{r_{C}+R},
$$

constants $\lambda_{1}, \lambda_{2}>0$,

$$
\begin{gathered}
\widehat{E}(t)=\eta_{1}(t)+\lambda_{1} v(t), \\
\widehat{i}(t)=\eta_{2}(t)+\lambda_{2} v(t) .
\end{gathered}
$$

The experimental results shown in this section satisfy condition (34) for global asymptotic stability (the boost power converter parameters, the saturation limits, and the selected numerical value of the gain $k_{a w}$ in (54) satisfy the condition for the function $\dot{\mathscr{W}}$ to be globally negative definite). 
In this case, the observation error dynamics are given by

$$
\frac{d}{d t}\left[\begin{array}{l}
z_{1} \\
z_{2}
\end{array}\right]=\left[\begin{array}{c}
-\frac{\lambda_{1}}{C}[1-u] \frac{R}{r_{C}+R} z_{2} \\
\frac{1}{L} z_{1}-\frac{r}{L} z_{2}-\frac{\lambda_{2}}{C}[1-u] \frac{R}{r_{C}+R} z_{2}
\end{array}\right],
$$

which is obtained from the boost converter equations in (1), the definition of $\mathbf{z}$ in (50), and the observer equations (62) and (64). It is possible to show that the state space origin of system (65) is exponentially stable.

6.3. Description of the Performed Experiments. The experimental results consist in comparing the KAO controller in (56) and (57) with the robust version of the new controller in (60), which uses the proposed observer in equations in (62) through the estimations $\widehat{E}(t)$ and $\widehat{i}(t)$.

In other words, in the experiments shown, the KAO controller (56) is based on observer (57) and (58), while the new controller (60) uses observer (62) and (64).

Notice that the controller proposed in [22] and described in (56) and (57) was designed under assumption $v(t)=v_{o}(t)$; that is to say, only the output voltage $v_{o}(t)$ is available for measurement. Motivated by this condition, we decided to implement the new scheme in (60), (62), and (64) assuming that $v(t)=v_{o}(t)$, so that both controllers are implemented in the same conditions.

For the implementation of the new scheme (60), (62), and (64) a measurement circuit for $v(t)$ could have been developed under knowledge of $r_{C}$. However, we decided to keep a fair comparison scenery.

6.4. Description of the Experimental Setup. An experimental platform has been built, featuring a PWM circuit with a switching frequency of $50[\mathrm{kHz}]$, a circuit board for measurements of the inductor current $i(t)$ and output voltage $v_{o}(t)$, including a NT-5 current sensor from FW Bell, and a boost converter circuit with exchangeable elements.

The controllers are programmed in the computational framework Simulink/Matlab with Real-Time Windows Target, installed in a PC with Windows XP. The latter hosts the data flowing through the data acquisition board DAQ6035E (inputs and outputs) by means of analog-to-digital and digital-to-analog conversions at up to $10[\mathrm{kHz}]$. This arrangement, represented in the form of a block diagram in Figure 4, allows implementing the controllers in real time.

Figure 5 shows a picture of the used experimental platform. A special circuit is used in order to vary the input voltage $E(t)$.

The parameters of the boost DC-DC power converter are the same as in Table 1. It should be noticed that the numerical values of the parasitic resistances $r_{L}$ and $r_{C}$ were obtained from rough estimations using a multimeter.

The experimental tests have been conducted in order to assess the performance of the scheme in (56) with respect to the new controller (60).

6.5. Results. For the implemented controllers, the limits of the saturation function were $u_{\min }=0.3$ and $u_{\max }=0.65$.

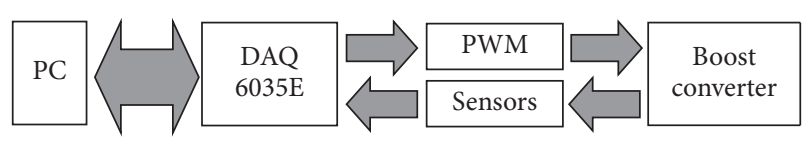

FIGURE 4: Block diagram on the representation of the experimental platform. The data acquisition board DAQ6035E is used for data transferring from the computer to the external circuits and vice versa.

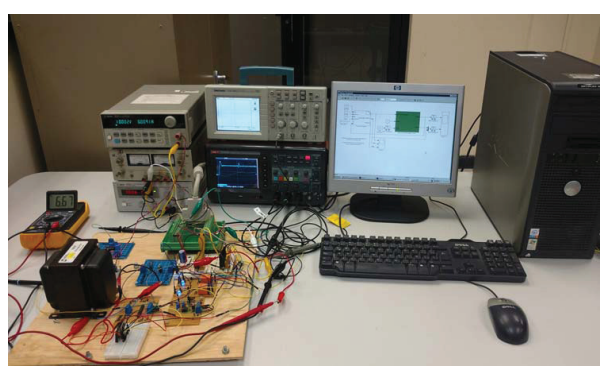

FIgURE 5: Experimental platform equipped with measurement circuits for output voltage and inductor current, circuit for online modification of the input voltage $E(t)$, PWM circuit, and boost converter with replaceable capacitor, inductor, and resistance.

Therefore, the duty cycle percentage remains bounded in the sense

$$
1-u_{\max }=0.35 \leq u(t) \leq 0.7=1-u_{\min } .
$$

For both schemes, we have considered that the boost converter is affected by a periodic time-varying supply voltage

$$
E(t)= \begin{cases}7[\mathrm{~V}] & \text { for } 0 \leq t<3 \\ 10[\mathrm{~V}] & \text { for } 3 \leq t<6\end{cases}
$$

All real-time experiments were carried out using the initial conditions $v(0)=6.65[\mathrm{~V}]$ and $i(0)=0.063[\mathrm{~A}]$ in the boost power converter.

Observer (57) is implemented using $\eta_{1}(0)=0[\mathrm{~V}]$ and $\eta_{2}(0)=0[\mathrm{~A}]$, and observer $(62)$ is implemented using $\eta_{1}(0)=$ $3.75[\mathrm{~V}]$ and $\eta_{2}(0)=0[\mathrm{~A}]$, which are safe initial conditions in the computing of $\widehat{D}^{*}(t)$. For all experiments, the observer gains $\lambda_{1}=0.5$ and $\lambda_{1}=0.1$ were used in observers (57) and (62).

The new controller in (60) was implemented with $\phi(0)=$ 0 and gains

$$
\begin{aligned}
\gamma & =10, \\
k_{a w} & =10 .
\end{aligned}
$$

Results obtained with the KAO controller and the new scheme are given in Figure 6, which depicts the output voltage $v_{o}(t)$, in Figure 7, which shows the time evolution of the duty cycle percentage $u(t)$, and in Figure 8, which illustrates the estimated supply voltage $\widehat{E}(t)$ for each scheme.

Better results are obtained with new scheme. More specifically, the settling time is much smaller for the new 
TABLE 2: Comparison of the settling times for the KAO controller (56) and the new controller (60).

\begin{tabular}{lccc}
\hline Time range & KAO controller (56) & New controller (60) & Improvement \\
\hline $0 \leq t<3$ & $0.369[\mathrm{~s}]$ & $0.096[\mathrm{~s}]$ & $73.98 \%$ \\
$3 \leq t \leq 6$ & $0.252[\mathrm{~s}]$ & $0.206[\mathrm{~s}]$ & $18.25 \%$ \\
\hline
\end{tabular}

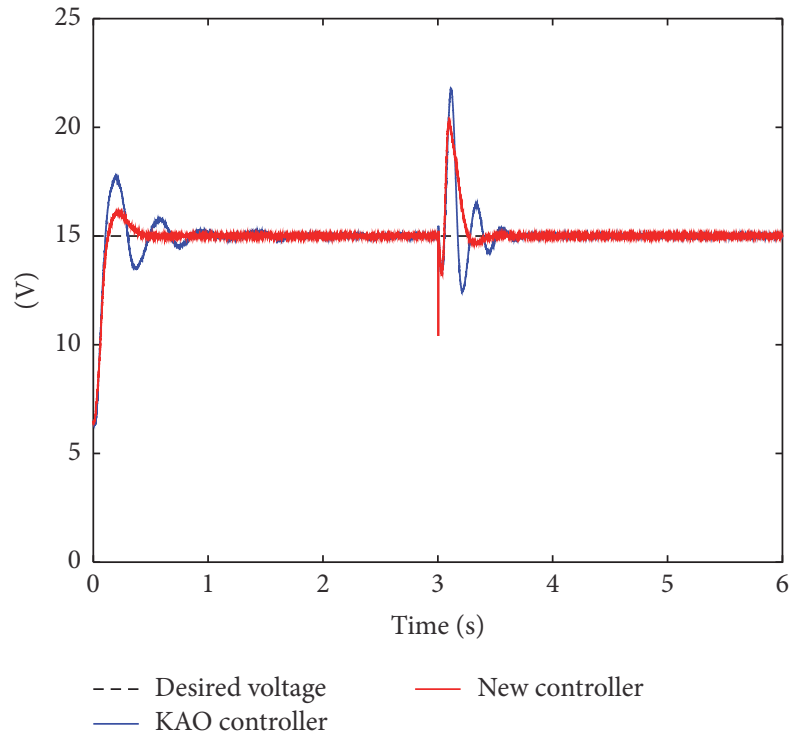

FIGURE 6: Experimental results: output voltage $v_{o}(t)$ obtained with the KAO controller (56) and for the new controller in (60).

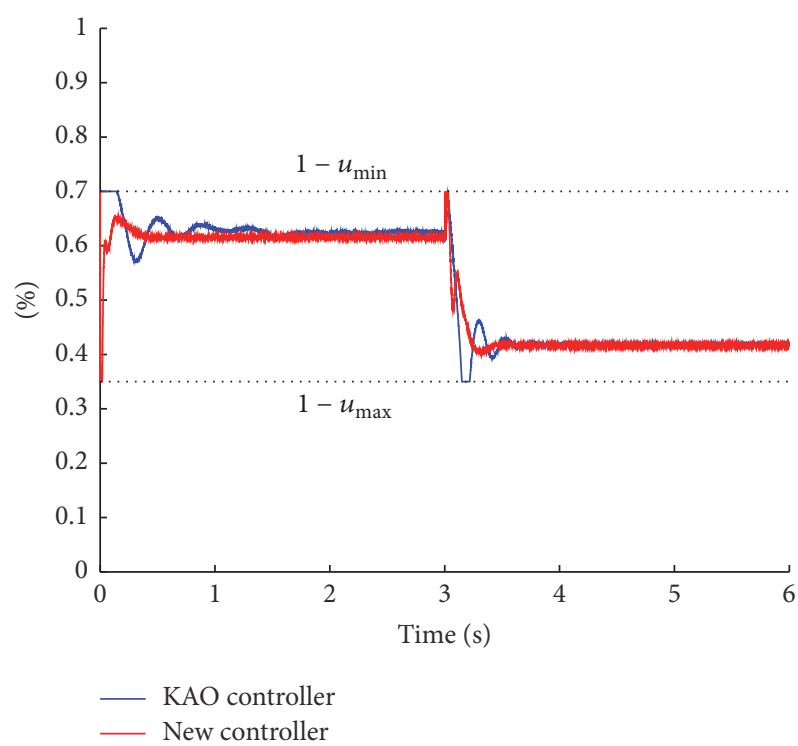

FIGURE 7: Experimental results: duty cycle percentage $u(t)$ obtained with the KAO controller (56) and for the new controller in (60).

scheme. See Table 2 for a comparison of the settling times for the controllers. Besides, as appreciated in Figure 6, the implementation of new controller presented lower voltage peaks during the transients at the beginning of the experiment and at the time the supply voltage $E(t)$ commutes from a value to the another.

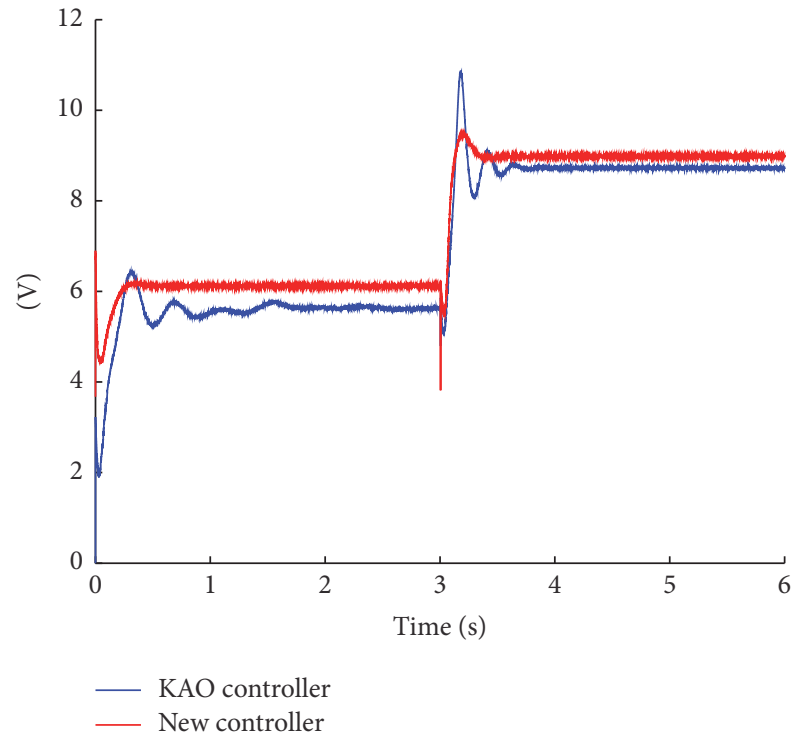

FIGURE 8: Experimental results: estimation of the supply voltage $\widehat{E}(t)$ obtained with the KAO controller (56) and with the new controller in (60).

It is important to observe that, despite the fact that control action $u(t)$ is saturated for short periods of time, the control objective of voltage regulation is satisfied by using both controllers, as predicted by theory.

The estimated input voltage $\widehat{E}(t)$ for both implementations does not converge exactly to the actual value of $E$. This is caused by the resistance present in the inductor, which is significative for high inductances. The value at which $\widehat{E}(t)$ converges is different from one controller to another. The main reason is that, in the new controller, observer (62) compensates the value of $r_{L}$, which results in a greater estimated supply voltage $\widehat{E}(t)$.

It is worthwhile to notice that the experimental platform uses a HUF75345G3 MOSFET that presents a static drainsource ON resistance $R_{\mathrm{DS}}$ equal to $0.006 \Omega$, which is considerably low in comparison to others switching semiconductors, such as the IRF640, which presents $R_{\mathrm{DS}}$ equal to $0.15 \Omega$. In this case, the reduction of $R_{\mathrm{DS}}$ value is approximately 25 times. The experimental platform uses the MBR1035 diode, which presents a low forward voltage, low power loss, and high efficiency features. These facts motivated us to neglect the drain-source ON resistance of the MOSFET and the diode resistance in the boost power converter model.

Since different components were tested, it should be stressed that the selection of the transistor and diode had a positive effect on the performance of evaluated controllers. 


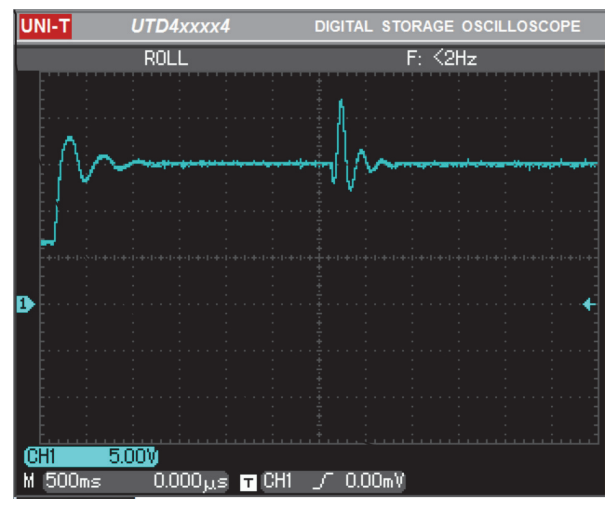

FIGURE 9: Experimental results: oscilloscope response to the output voltage $v_{o}(t)$ of the KAO controller (56).

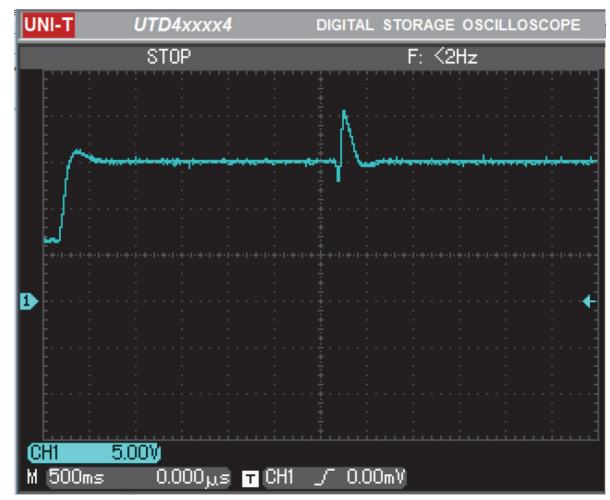

FIGURE 10: Experimental results: oscilloscope response to the output voltage $v_{o}(t)$ of the new controller $(60)$.

However, the new control scheme presented the best regulation performance.

Moreover, given that the data is being collected at $10[\mathrm{kS} / \mathrm{s}]$ by the specified DAQ device, an UNI-T UTD4204C desktop digital storage oscilloscope was used to observe the output voltage $v_{o}(t)$ for comparison purposes. See Figures 9 and 10 for the implementation the KAO controller (56) and the new controller (60), respectively. Results are the same as the ones displayed in Figure 6.

\section{Conclusions}

The problem of voltage regulation under input saturation was addressed in this paper. Special attention was payed to the DC-DC boost converter model, which considered the losses due to parasitic effects in the inductor and capacitor. The introduced controller is theoretically supported by a rigorous stability analysis, which is based on Lyapunov's theory. A tuning guideline was derived, which guarantees global asymptotic stability. Besides, the problem of uncertain supply voltage and unmeasurable current was addressed. Based on known results in literature, a new observer-based controller was proposed. The new schemes were tested by using numerical simulations and real-time experiments presenting better performance with respect to other algorithms.

\section{Competing Interests}

The authors declare that there is no conflict of interests regarding the publication of this paper.

\section{Acknowledgments}

This work was supported by Secretaría de Investigación y Posgrado del IPN and CONACyT Project no. 176587, Mexico.

\section{References}

[1] B. K. Bose, Ed., Modern Power Electronics: Evolution, Technology, and Applications, IEEE Press, New York, NY, USA, 1992.

[2] R. Ortega, A. Loría, P. J. Nicklasson, and H. Sira-Ramirez, Pasivity-Based Control of Euler-Lagrange Systems, Springer, London, UK, 1998.

[3] H. Sira-Ramirez and R. Silva-Ortigoza, Control Design Techniques in Power Electronics Devices, Springer, London, UK, 2006.

[4] B. L. Dokić and B. Blanuša, Power Electronics-Converters and Regulators, Springer, Berlin, Germany, 2015.

[5] F. Bonanno, G. Capizzi, and G. Lo Sciuto, "Improved SMPS modeling for photovoltaic applications by a novel neural paradigm with Hamiltonian-based training algorithm," in Proceedings of the 5th International Conference on Clean Electrical Power (ICCEP '15), pp. 723-730, Taormina, Italy, June 2015.

[6] H. Du, X. Lai, and C. Liu, "Design of a synchronous boost DC-DC converter with constant current mode control in MPP," Analog Integrated Circuits and Signal Processing, vol. 84, no. 2, pp. 223-235, 2015.

[7] K. Mohanraj, C. Danya Bersis, and S. Sekhar Dash, "Simulation of open loop and feed-back controlled bridgeless PFC boost converter," in Power Electronics and Renewable Energy Systems, pp. 29-38, Springer, New Delhi, India, 2015.

[8] A. Tani, M. B. Camara, and B. Dakyo, "Energy management based on frequency approach for hybrid electric vehicle applications: fuel-cell/lithium-battery and ultracapacitors," IEEE Transactions on Vehicular Technology, vol. 61, no. 8, pp. 33753386, 2012.

[9] J. Alvarez-Ramirez, G. Espinosa-Perez, and D. Noriega-Pineda, "Current-mode control of DC-DC power converters: a backstepping approach," International Journal of Robust and Nonlinear Control, vol. 13, no. 5, pp. 421-442, 2003.

[10] C. Olalla, I. Queinnec, R. Leyva, and A. El Aroudi, "Robust optimal control of bilinear DC-DC converters," Control Engineering Practice, vol. 19, no. 7, pp. 688-699, 2011.

[11] M. Spinetti, E. Fossas, and D. Biel, "Stabilitiy analysis of a Lyapunov-based controlled boost converter," in Proceedings of the 48th IEEE Conference on Decision and Control held jointly with 2009 28th Chinese Control Conference (CDC/CCC '09), pp. 6544-6548, Shanghai, China, December 2009.

[12] Z. Wu and C.-H. Liu, "Nonlinear current-mode controller for DC/DC boost power converters," Electronics Letters, vol. 47, no. 3, pp. 209-211, 2011.

[13] Y. Massaoudi, D. Elleuch, D. Mehdi, T. Damak, and G. Hashim, "Comparison between non linear controllers applied to a DC-DC boost converter," International Journal of Innovative Computing, Information \& Control, vol. 11, no. 3, pp. 935-947, 2015. 
[14] H. Cho, S. J. Yoo, and S. Kwak, "State observer based sensor less control using Lyapunov's method for boost converters," IET Power Electronics, vol. 8, no. 1, pp. 11-19, 2015.

[15] J.-J. E. Slotine and W. Li, Applied Nonlinear Control, PrenticeHall, Englewood Cliffs, NJ, USA, 1991.

[16] C. Olalla, R. Leyva, A. El Aroudi, I. Queinnec, and S. Tarbouriech, "Ho control of DC-DC converters with saturated inputs," in Proceedings of the 35th Annual Conference of the IEEE Industrial Electronics Society (IECON '09), pp. 548-553, Porto, Portugal, November 2009.

[17] M. Feki, A. El Aroudi, B. G. M. Robert, and N. Derbel, "Control of a two-cell $\mathrm{dc} / \mathrm{dc}$ converter in presence of saturating duty cycle," in Proceedings of the IEEE 13th Power Electronics and Motion Control Conference (EPE-PEMC '08), pp. 2120-2125, IEEE, Poznań, Poland, September 2008.

[18] Q. Chen, X. Ren, and J. A. Oliver, "Identifier-based adaptive neural dynamic surface control for uncertain DC-DC buck converter system with input constraint," Communications in Nonlinear Science and Numerical Simulation, vol. 17, no. 4, pp. 1871-1883, 2012.

[19] A. Benzaouia, H. M. Soliman, and A. Saleem, "Regional pole placement with saturated control for DC-DC buck converter through Hardware-in-the-Loop," Transactions of the Institute of Measurement and Control, vol. 38, no. 9, pp. 1041-1052, 2016.

[20] G. C. Konstantopoulos and Q. Zhong, "Nonlinear control of $\mathrm{dc} / \mathrm{dc}$ power converters with inherent current and power limitation," in Proceedings of the 24th Mediterranean Conference on Control and Automation (MED '16), pp. 949-954, Athens, Greece, June 2016.

[21] J. Guzmán-Guemez and J. Moreno-Valenzuela, "Saturated control of boost DC-to-DC power converter," Electronics Letters, vol. 49, no. 9, pp. 613-615, 2013.

[22] D. Karagiannis, A. Astolfi, and R. Ortega, "Two results for adaptive output feedback stabilization of nonlinear systems," Automatica, vol. 39, no. 5, pp. 857-866, 2003.

[23] Y. Yao, F. Fassinou, and T. Hu, "Stability and robust regulation of battery-driven boost converter with simple feedback," IEEE Transactions on Power Electronics, vol. 26, no. 9, pp. 2614-2626, 2011.

[24] C. Yfoulis, D. Giaouris, F. Stergiopoulos, C. Ziogou, S. Voutetakis, and S. Papadopoulou, "Robust constrained stabilization of boost DC-DC converters through bifurcation analysis," Control Engineering Practice, vol. 35, pp. 67-82, 2015.

[25] M. Krstić, I. Kanellakopoulos, and P. V. Kokotović, Nonlinear and Adaptive Controller Control Design, John Wiley and Sons, New York, NY, USA, 1995.

[26] H. K. Khalil, Nonlinear Systems, Prentice-Hall, Upper Saddle River, NJ, USA, 2nd edition, 1996.

[27] A. Loría and J. De León Morales, "On persistently exciting observers and a non-linear separation principle: application to the stabilization of a generator," International Journal of Control, vol. 76, no. 6, pp. 607-617, 2003. 


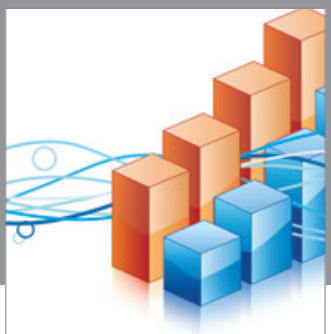

Advances in

Operations Research

vatem alat4

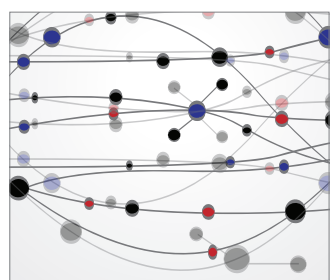

\section{The Scientific} World Journal
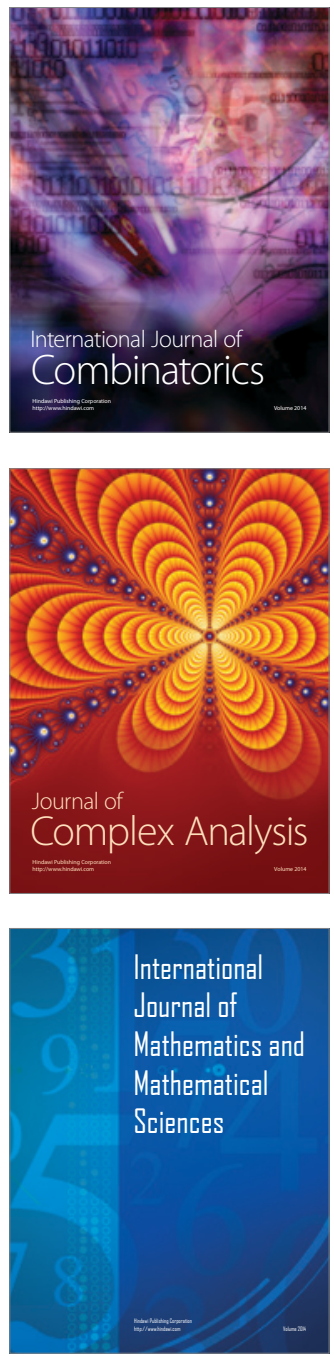
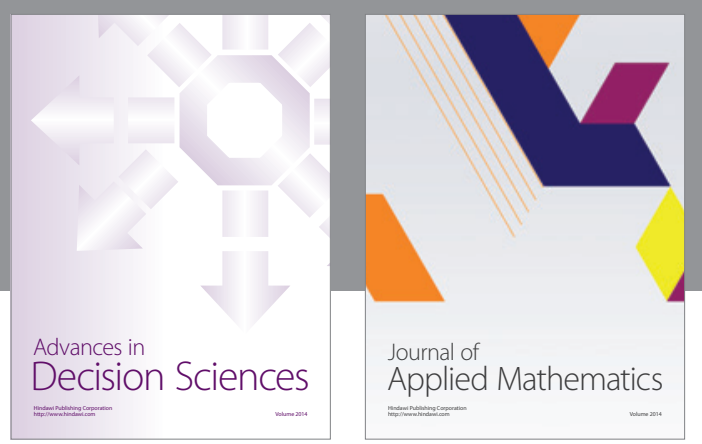

Algebra

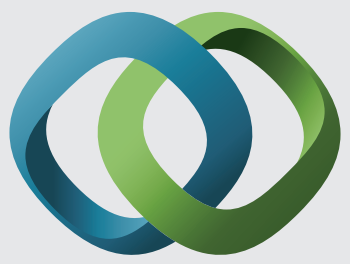

\section{Hindawi}

Submit your manuscripts at

https://www.hindawi.com
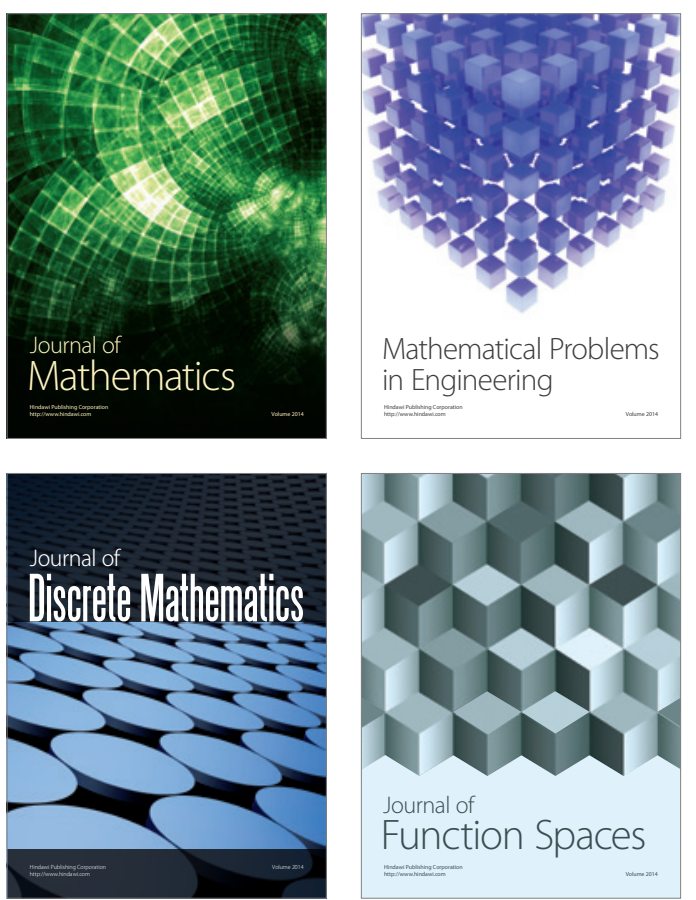

Mathematical Problems in Engineering
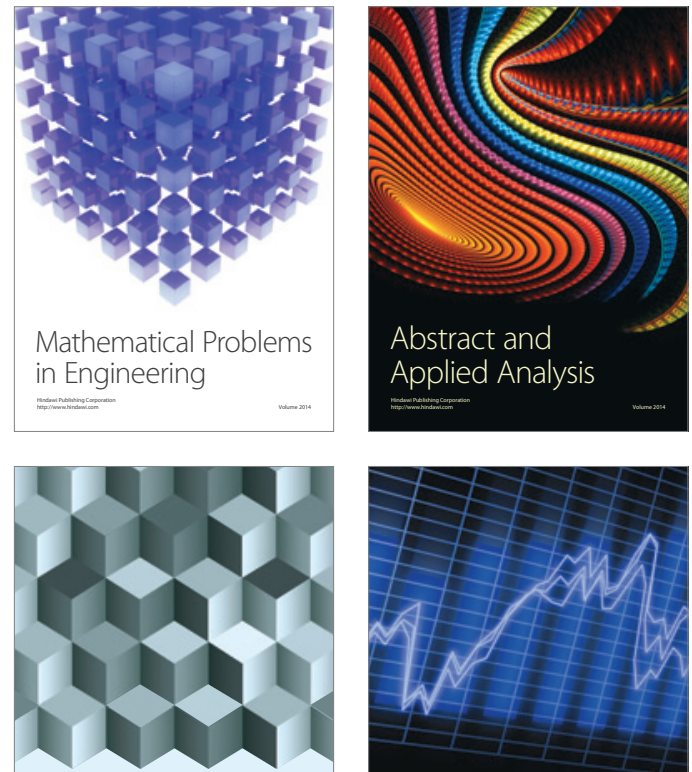

Journal of

Function Spaces

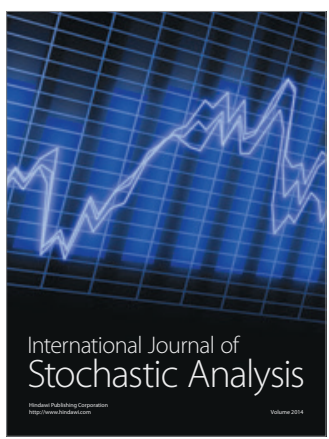

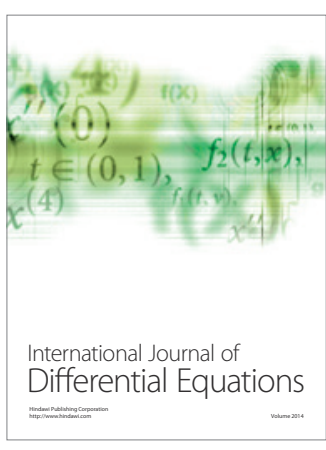
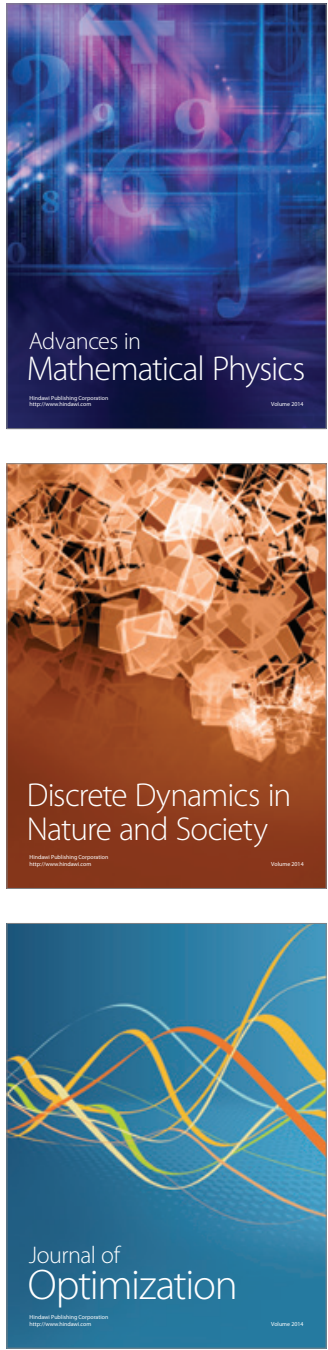